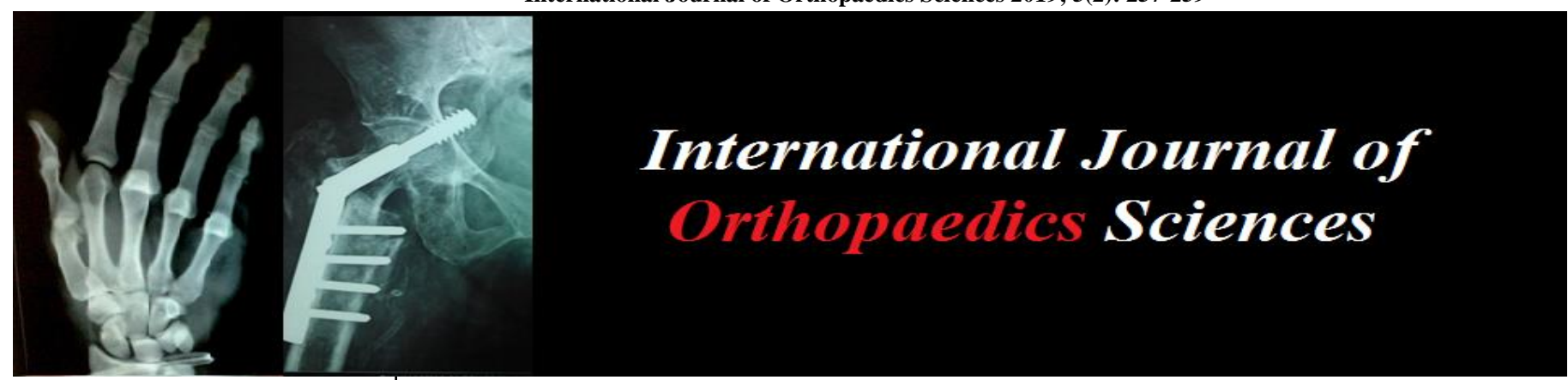

ISSN: $2395-1958$

IJOS 2019; 5(2): 257-259

(C) 2019 IJOS

www.orthopaper.com

Received: 04-02-2019

Accepted: 08-03-2019

Yasser Alwabli

MBBS, SB-Orth, Board-certified

Orthopedic Surgeon, Qassim

University Medical School,

Department of Orthopedic

Surgery, Qassim, Saudi Arabia

\section{A rare presentation of a bilateral tear of the posterior horn of medial meniscus in a young patient: A case report}

\section{Yasser Alwabli}

DOI: https://doi.org/10.22271/ortho.2019.v5.i2f.32

\section{Abstract}

Introduction: There are two menisci in the human knee, the medial and lateral meniscus. The function of the menisci is shock absorption, proprioception, and improved stability. The vascular supply of the meniscus is crucial as it dictates the choice between surgical options. Meniscal tears are common and account for the majority of surgical knee procedures. The tear usually affects one knee and bilateral meniscal tears are extremely uncommon. Treatment of meniscal tears can be non-operative or operative. Operative treatment is indicated specifically if the tear is associated with mechanical symptoms such as locking or catching.

Clinical case: A 20-year-old male patient presented at our university out-patient clinic with bilateral knee pain after a soccer injury a few months prior. A detailed history and physical examination raised a suspicion of bilateral meniscal tears. Advanced imaging including magnetic resonance imaging (MRI), confirmed the diagnosis. After discussion with the patient and explaining different treatment options, non-operative treatment was carried out in the form of a physical therapy program at our center. On follow up, the patient showed significant improvement of his symptoms.

Conclusion: Bilateral meniscal tears are extremely uncommon injuries with only a few reported cases in the literature. The reported cases were bucket-handle type tears which were treated arthroscopically. In our case, there were no mechanical symptoms and the patient improved with non-operative treatment.

Keywords: knee meniscus, bilateral meniscus tear, posterior horn, partial meniscectomy

\section{Introduction}

There are two menisci in the human knee and are named according to their position, the medial and lateral meniscus. The menisci play important roles in the knee such as shock absorption and proprioception. Knee stability is another important function of the meniscus [1]. The medial meniscus is c-shaped and thicker posteriorly, which promotes greater posterior stabilization of the knee. The lateral meniscus is semicircular in shape and covers a larger area of the tibial plateau compared to the medial meniscus.

The vascularity of the menisci is extremely important as it will dictate whether the torn meniscus is repairable surgically. Although some authors have proposed a complex vascular categorization of the meniscus vascularity ${ }^{[2]}$, the commonly used vascular classification is whether the tear is in the red (peripheral), red-white (middle), or white zone (inner) ${ }^{[3]}$ of the meniscus. Therefore, tears in the red or red-white zones have a higher chance of healing and hence are repairable, while those in the white zone are irreparable and typically are treated with a partial meniscectomy.

Meniscal tears are common injuries, accounting for 61 per 100,000 individuals in the general population ${ }^{[4]}$. Meniscal tears can be acute traumatic or degenerative tears ${ }^{[5]}$. In young patients the tears are commonly traumatic due to sport injuries, while in older patients tears are usually degenerative in nature. The types of torn meniscus include horizontal, bucket-handle, longitudinal, oblique, radial, and complex (a combination of different tear types). The tear type and location are reliable clinical and surgical indicators for the healing potential of a torn meniscus. This is an important element in clinical assessment as it aids in surgical decisionmaking and treatment option prioritization.

Diagnosis of meniscal tears is typically clinical. The differential diagnosis of a meniscal injury
Yasser Alwabli

MBBS, SB-Orth, Board-certified

Orthopedic Surgeon, Qassim

University Medical School,

Department of Orthopedic

Surgery, Qassim, Saudi Arabia 
should be a top priority for patients presenting with knee pain [4]. Also, symptoms of catching and locking are not uncommon ${ }^{[3]}$. Physical examination of the knee will reveal joint line tenderness with a palpable click or snap ${ }^{[6]}$. Joint line tenderness has over $70 \%$ sensitivity and up to $90 \%$ specificity for meniscal tears ${ }^{[3]}$. Several special clinical tests have been described for the accurate diagnosis of meniscal tears, for example McMurray's test and the Apley test. However a new test, the Thessaly test, has shown low rates of false-negative and false-positive results ${ }^{[6]}$. This test can be performed by asking the patient to stand on the affected knee and flex it to 20 degrees while the patient twists the knee with an internal and external rotation.

Although radiographs are routinely obtained on initial clinical evaluation of any patient with knee pain, radiographs of meniscal tear cases should be normal on regular x-rays. In addition to a clinical evaluation which includes special tests, magnetic resonance imaging (MRI) is the most sensitive and specific imaging modality to confirm a diagnosis of meniscal tears. An MRI is routinely obtained in suspected cases of meniscal tears for diagnosis confirmation and treatment planning.

The treatment of meniscal tears can be operative or nonoperative. The decision on appropriate treatment options for a given patient depends on many factors including age, comorbidities, compliance, and tear charecteristics ${ }^{[7]}$. Nonoperative treatment can be utilized specifically for degenerative tear types. However, even with traumatic tears conservative treatment can be utilized if no mechanical symptoms are present. Operative treatment is the primary treatment whenever there are mechanical symptoms. A partial meniscectomy and meniscal repair are surgical options and are largely based on tear characteristics ${ }^{[7]}$.

Meniscal tears usually affect one knee and rarely affect both knees simultaneously. This case report describes a rare presentation of a young patient with bilateral meniscus tears in both knees.

\section{Clinical case}

A 20-year-old male patient, a university student and recreational soccer player with no significant medical history, presented at our university orthopedic out-patient clinic with a chief complaint of bilateral knee pain. He also complained of a crepitus sensation, mainly in the right knee. On further questioning, the patient had a soccer injury to his knees a few months prior, and had developed these complaints since then. There were no mechanical symptoms. Other elements of the history were unremarkable. Physical examination revealed a healthy young patient with normal vital signs and a normal body mass index (BMI). Local examination to the knee showed a full range of motion for both knees. However, joint line tenderness was elicited upon palpation over the joint line on the medial side of both knees. X-rays were obtained and returned as normal. An MRI was performed on both knees to rule out intra-articular pathology. The MRI of both knees was reported as follows: in the right knee the multiplanar MRI of the sagittal, coronal, and transverse planes using T1 weighted and $\mathrm{T} 2$ weighted sequences showed intact anterior cruciate ligament (ACL), posterior cruciate ligament (PCL), patellar tendon, and lateral meniscus. The medial meniscus showed a tear in the posterior horn reaching the inferior articular surface. In the left knee, the multiplanar MRI of the sagittal, coronal, and transverse planes using T1 weighted and T2 weighted sequences showed intact ACL, PCL, patellar tendon, and lateral meniscus. The medial meniscus showed an oblique tear in the posterior horn of the meniscus.

We discussed the diagnosis and MRI findings with the patient and offered treatment options including non-operative treatment. A decision for non-operative treatment was made and a physical therapy program was prescribed, which was performed at our physical therapy center. On both the oneand three-month follow ups, the patient showed less pain in both knees and relief of the crepitus sensation. Surgical treatment continued to be an option if there was a change in the clinical picture and most importantly, if the patient developed mechanical symptoms. However, the patient showed significant improvement in his symptoms and elected to continue with non-operative treatment.

\section{Discussion}

The presentation of bilateral meniscus tears is extremely rare. Upon literature review, there are only a few reported cases of bilateral tears, which are mainly bucket-handle type ${ }^{[8,10]}$. In cases of sport injuries, especially with a twisting of the knee, suspicion of meniscal tears is warranted. Similarly, other knee structures such as the ACL and PCL should be kept in mind when dealing with knee injuries. A clinician should take a detailed history, conduct a physical examination and, if needed, perform advanced imaging to reach to the correct diagnosis ${ }^{[11]}$. Once the diagnosis has been established a proper treatment plan can be designed, taking into consideration patient factors and tear characteristics ${ }^{[7]}$.

The mainstay of treatment of acute traumatic meniscal injuries is surgical, particularly if associated with mechanical symptoms such as locking or catching. In cases of buckethandle tears, treatment is almost always surgical through arthroscopic meniscal repair andlor partial meniscectomy. This was the treatment performed in the cases reviewed ${ }^{[8-10]}$. However, our case was not associated with mechanical symptoms and was not a bucket-handle tear type. Accordingly, and after discussion with the patient a nonoperative treatment was carried out. This form of treatment gave the patient greater symptomatic relief and better function than during the pre-treatment period. As previously mentioned, the treatment of meniscal tears is based on many factors, including patient-related factors and tear characteristics.

\section{Conclusion}

Meniscal tears are common injuries and are often indicators for knee surgery. Patients with knee pain after sport injuries should be worked up to rule out meniscal tears. In very rare cases of bilateral meniscal tears, non-operative treatment is a valid option and may be prescribed if it is the patient's preference and there is no clear indication for surgical treatment.

\section{References}

1. Torres SJ, Hsu JE, Mauck RL. Meniscal Anatomy. In: Kelly IV JD, ed. Meniscal Injuries: Management and Surgical Techniques. New York, NY: Springer New York. 2014, 1-7. Doi: 10.1007/978-1-4614-8486-8_1.

2. Cooper DE, Arnoczky SP, Warren RF. Meniscal repair. Clin Sports Med. 1991; 10(3):529-548.

3. Laible C, Stein DA, Kiridly DN. Meniscal Repair. J Am Acad Orthop Surg J Am Acad Orthop Surg. 2013; 21(4):204-213.

4. McHale KJ, Park MJ, Tjoumakaris FP. Physical Examination for Meniscus Tears. In: Kelly IV JD, ed. Meniscal Injuries: Management and Surgical Techniques. 
New York, NY: Springer New York; 2014, 9-20. Doi: 10.1007/978-1-4614-8486-8_2.

5. Poehling GG, Ruch DS, Chabon SJ. The landscape of meniscal injuries. Clin Sports Med. 1990; 9(3):539-549.

6. Karachalios T, Hantes M, Zibis AH, Zachos V, Karantanas AH MK. Diagnostic accuracy of a new clinical test (the Thessaly test) for early detection of meniscal tears. J Bone Jt Surg Am. 2013; 343:24.

7. Mordecai SC. Treatment of meniscal tears: An evidencebased approach. WJO World J Orthop. 2014; 5(3):233.

8. Sezer HB, Arikan Y, Armagan R, Eren OT. Bilateral bucket handle medial meniscal tears of the knee: A case report. IJSCR Int J Surg Case Reports. 2013; 4(10):926928.

9. Walker B, Limbert A. Bilateral, simultaneous medial meniscus bucket handle tears in a 23-year-old female. Case Rep Orthop, 2014.

10. Abbott J, Lam KS, Srivastava VM, Moulton A. Sand pits and bucket handles: A case of bilateral traumatic buckethandle tears of the medial menisci. YJARS Arthrosc J Arthrosc Relat Surg. 2000; 16(6):1-3.

11. Lefevre N, Naouri JF, Herman S, Gerometta A, Klouche S, Bohu Y. A Current Review of the Meniscus Imaging: Proposition of a Useful Tool for Its Radiologic Analysis. Radiol Res Pract. 2016; 8329296. doi:10.1155/2016/8329296. 